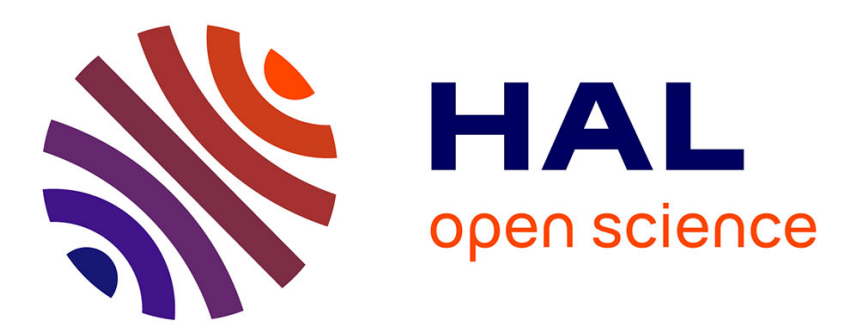

\title{
Multiphoton microscopy for the in-situ-investigation of cellular processes and integrity in cryopreservation
}

Daniel Doerr, Martin Stark, Friederike Ehrhart, Heiko Zimmermann, Frank Stracke

\section{- To cite this version:}

Daniel Doerr, Martin Stark, Friederike Ehrhart, Heiko Zimmermann, Frank Stracke. Multiphoton microscopy for the in-situ-investigation of cellular processes and integrity in cryopreservation. Biotechnology Journal, 2009, 4 (4), pp.1215-n/a. 10.1002/biot.200800212 . hal-00477771

\section{HAL Id: hal-00477771 https://hal.science/hal-00477771}

Submitted on 30 Apr 2010

HAL is a multi-disciplinary open access archive for the deposit and dissemination of scientific research documents, whether they are published or not. The documents may come from teaching and research institutions in France or abroad, or from public or private research centers.
L'archive ouverte pluridisciplinaire HAL, est destinée au dépôt et à la diffusion de documents scientifiques de niveau recherche, publiés ou non, émanant des établissements d'enseignement et de recherche français ou étrangers, des laboratoires publics ou privés. 


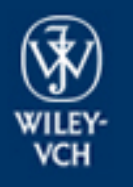

Biotechnology Journal

\section{Multiphoton microscopy for the in-situ-investigation of cellular processes and integrity in cryopreservation}

\begin{tabular}{|r|l|}
\hline Journal: & Biotechnology Journal \\
\hline Manuscript ID: & BIOT-2008-0212.R2 \\
\hline Wiley - Manuscript type: & Technical Report \\
\hline Author: & 12-Feb-2009 \\
\hline & $\begin{array}{l}\text { Complete List of Authors: } \\
\text { Eraunherer IBMT } \\
\text { Zimmermann, Heiko; Fraunhofer IBMT, Cryobiotechnology } \\
\text { Stracke, Frank; FhG IBMT, Biomedical Optics }\end{array}$ \\
\hline Keywords: Fraunhofer IBMT, Cryobiotechnology & $\begin{array}{l}\text { cryo-preservation, cryo-microscopy, multiphoton microscopy, FLIM, } \\
\text { cryo-injury }\end{array}$ \\
\hline
\end{tabular}

\section{scholaroNE" \\ Manuscript Central}


Technical Report

\title{
Multiphoton microscopy for the in-situ-investigation of cellular processes and integrity in cryopreservation
}

\author{
Daniel Doerr ${ }^{1}$, Martin Stark ${ }^{1}$, Friederike Ehrhart ${ }^{1}$, Heiko Zimmermann ${ }^{1,2}$, \\ Frank Stracke ${ }^{1}$ \\ 1) Fraunhofer Institut Biomedizinische Technik IBMT, Ensheimer Str. 48, 66386 St. \\ Ingbert, Germany \\ 2) Molekulare \& Zelluläre Biotechnologie \& Nanotechnologie, Universität des \\ Saarlandes, Campus 66123 Saarbrücken, Germany
}

Keywords: cryo-preservation, cryo-microscopy, multiphoton microscopy, FLIM, native fluorescence

\section{Corresponding author:}

Dr. Frank Stracke

Fraunhofer Institute Biomedical Technology,

Ensheimer Str. 48

D-66386 St. Ingbert

0049-6894981165

0049-6894981400 
frank.stracke@ibmt.fhg.de

\begin{abstract}
In this study we demonstrate a new non-invasive imaging method for monitoring freezing processes in biological samples and to investigate life in the frozen state. It combines an laser scanning microscope with a computer-controlled cryo-stage. Near infrared femtosecond laser pulses evoke the fluorescence of endogenous fluorophores and fluorescent labels due to multiphoton absorption. The inherent optical nonlinearity of multiphoton absorption allows 3D fluorescence imaging for optical tomography of frozen biological material in situ. As an example for functional imaging we use fluorescence lifetime imaging (FLIM) to create images with chemical and physical contrast.
\end{abstract}


Abbreviations

\begin{tabular}{ll} 
FLIM & fluorescence lifetime imaging \\
FWHM & full width (at) half maximum \\
\hline IRF & instrument response function \\
NAD $(P) H$ & nicotineamide adenine dinucleotide \\
& (phosphate), reduced form \\
NIR & near infrared \\
TCSPC & time correlated single photon counting \\
Vis & visible spectral range
\end{tabular}


Cryopreservation is currently the only widely applicable technique for the long-term storage of cells and other biological specimens without deterioration of their vital functions. There is rapid growth of specimen numbers in the fields of reproductive medicine [1]; cancer treatment; regenerative medicine and tissue engineering [2]; stem cells [3] and the storage of biopsies. This increases demands for suitable tools for quality control during preservation, for optimizing freeze/thaw protocols and for understanding the underlying mechanisms. The present work is focused on freezing of bio-specimens for reanimation purposes. Freezing applications beyond this scope, as e.g. snapshots of the biochemical status of a cell for subsequent chemical analysis or biomarker investigations on tissue specimens, may also benefit from the ability of this technique to reveal morphological and chemical changes during the freezing process.

Almost every cell line and biomaterial has different optimal freezing procedures depending on cell specific properties like hydraulic membrane conductance, osmotic active and inactive volumes and cytoplasm composition [4]. This leads to a vast array of freezing protocols for different cells to be found in literature. There is no comprehensive study in the correlations between many cellular characteristics and optimal freezing parameters. A mechanistic investigation on freezing injury upon different cooling rates including 
some very diverse cell types is given in [5], Considerable effort is needed to optimise freezing rate, medium composition, cryoprotectant agents and containers for a novel type of sample. Currently, such investigations are predominantly empirical and based on the determination of survival rates after a freeze/thaw cycle [6][7][8], We need in situ observation techniques with subcellular resolution to understand the cellular processes during freezing, cryostorage and thawing.

Optical transmission microscopy is frequently used [9][10] but does not provide three dimensional resolution and is limited by strong light scattering by the micro-domains and the need for thermal isolation. This technique was established in the 1830's by Goeppert for observing plant cell behaviour upon freezing [13], Today's state of development reflects the methodology of the 1970s, when Diller developed the first computer controlled cryo-stage for microscopic applications [14]). Cryo-electron microscopy or tomography after cryo-sectioning [11][12] provides 3D morphology with resolution in the nanometer range. But the elaborate specimen preparation precludes in situ studies and dynamic applications. Confocal laser scanning microscopy [15] allows 3D observations in situ, but is also limited by scattering and carries the risk of phototoxic side effects.

Here we report on the introduction of multiphoton laser scanning microscopy into cryo-biotechnology. This is a fluorescence
Deleted: [5]

Deleted: [4]

Deleted: [6]
Deleted: [5]
Deleted: [7]
Deleted: [6]
Deleted: [8]
Deleted: [7]
Deleted: [9]
Deleted: [8]
Deleted: [10]
Deleted: [9]

Deleted: [13]

Deleted: [12]

\begin{tabular}{|l|}
\hline Deleted: [14] \\
\hline Deleted: [13] \\
\hline Deleted: [11] \\
\hline Deleted: [10] \\
\hline Deleted: [12] \\
\hline Deleted: [11] \\
\hline
\end{tabular}

Deleted: [15]

Deleted: [14] 
imaging technique with resolution benchmarks similar to confocal microscopy, but with significantly reduced scattering limitations and phototoxic interference. There are two reasons for the low susceptibility of multiphoton microscopy to sample scattering.

Firstly, fluorescence is excited by the simultaneous absorption of two (or more) near infrared (NIR) photons. Each photon has half the energy (double the wavelength) of a single ultraviolet or visible (UV/Vis) photon matching the transition energy. Since scattering declines_strongly with wavelength the doubling of the illumination wavelength drastically reduces scattering losses in excitation direction. In addition most samples show virtually no absorption in the NIR. Therefore the penetration depth (distance into a specimen at which $\mathrm{e}^{-1}$ of incident photons remain unscattered) in two photon excitation is many times greater than in linear (single photon) excitation (scheme 1a) in the ice matrix and in biological matter.

Secondly, excitation probability varies non-linearly with illumination intensity (squared for two-photon excitation, cubed for three). Therefore fluorophores are excited essentially only in the subfemtoliter focal volume and each fluorescence photon arriving at the detector can unambiguously be attributed to this volume. 3D resolution on the $\mu \mathrm{m}$-scale is thus an inherent feature of focused multiphoton excitation, and no spatial filter (pinhole) is necessary. This allows the wide-field collection of fluorescence light and, in 
contrast to confocal imaging, scattered fluorescence photons can be detected (scheme 1b). There are virtually no endogenous absorbers in biomaterials in the spectral region between $700 \mathrm{~nm}$ and $1000 \mathrm{~nm}$ (termed optical window in biomedical imaging). This lack of endogenous one-photon-absorbers in the NIR means there is very little out-of-focus phototoxicity in multiphoton microscopy.

The enormous focal intensities on the order of $\mathrm{GW} / \mathrm{cm}^{2}$ needed for significant two photon excitation are provided by an ultra-shortpulsed titanium:sapphire-laser (Chameleon Ultra II, Coherent, Santa Clara, USA) efficiently focused by microscopy objectives. Image reconstruction is carried out by $x y$-beam scanning using commercial laser scanning microscopes coupled to a temperature-controlled cryo-stage (MDS 600, Linkam, Waterfield, UK). Two different setups were used: (1) For observations down to liquid nitrogen temperatures an upright laser scanning microscope (Femt-O-Cut, JenLab GmbH, Jena, Germany) was equipped with a 50× / 0.5 NA objective without immersion medium. This setup provides good thermal insulation and a large working distance, but has only moderate spatial resolution $\underline{(F W H M(x) \approx 0,7 \mu \mathrm{m})}$. (2) For high resolution images an inverted laser scanning microscope (Zeiss Axiovert LSM 510 META NLO, Carl Zeiss Jena, Germany) equipped with an objective inverter (LSMTech,

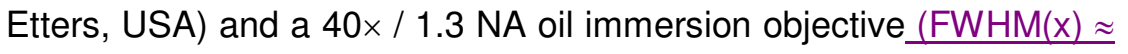
$\underline{0,3 \mu \mathrm{m})}$. The thermal connection between sample and objective does 
not allow for temperatures beyond $-100{ }^{\circ} \mathrm{C}$. The working distance of the immersion objective is furthermore limited to $200 \mu \mathrm{m}$.

Fluorescence lifetime imaging (FLIM) was performed using the time correlated single photon counting method [16][17], A SPC 730 FLIM module and a PMC 100 detector (both Becker \& Hickl, Berlin, Germany) were used for the fluorescence decay measurements. A detailed description of the FLIM detection setup can be found elsewhere [18], For a general introduction into applications and instrumentation of FLIM the reader is referred to [19], and to [20] for a brief introduction into the molecular fundamentals of this technique.

Different types of biological samples were used to demonstrate the capabilities of multiphoton cryo-microscopy. L929 cells (DSMZ, Braunschweig, Germany) were cultured according to the standard protocols. L929 cells were displaced from the culture flasks and resuspended with culture medium. Dimethyl sulfoxide (DMSO, Wacker Chemie AG, Germany) was optionally used as cryoprotectant at $10 \%$ (by mass) in the freezing medium. It was added to the cell suspension and incubated for $30 \mathrm{~min}$ at $4{ }^{\circ} \mathrm{C}$ before freezing. To analyze adherent cells, the L929 cells were cultivated for one day on glass cover slips. Cell suspensions and adherent cells were directly transferred to the sample chamber of the freezing unit. Freshly collected leaves of Elodea densa were transferred to the sample chamber and immersed in water for the multiphoton cryo- 
FLIM studies. Information on cooling rates and media of the experiments are given in the individual descriptions. Endogenous contrast arises from fluorescent compounds of the specimens, as e.g. $\mathrm{NAD}(\mathrm{P}) \mathrm{H}$ and flavins of the cellular mitochondria $[21]$ as well as Deleted: [21] lignin and chlorophyll of the Elodea densa. These fluorophores can easily be excited by the same NIR-wavelength in multiphoton microscopy, whereas in particular $\mathrm{NAD}(\mathrm{P}) \mathrm{H}$ and the flavins require UV-illumination in single-photon-excitation. Artificial labelling of cellular compartments was carried out with calcein (cytoplasm) and Hoechst 33342 (nuclei) (Molecular Probes, Eugene, USA) on vital cells.

The major challenge for optical microscopy of cryo-preserved samples is the ice crystal formation in the frozen state, extracellular as well as intracellular. Due to strong scattering and reflection of the ice crystals, only blurred micrographs with no access to cellular details are possible in traditional wide field cryo-microscopy. Scheme 1 compares different imaging modalities for the same specimens, showing the improvements in contrast and spatial resolution in multiphoton microscopy. For the microscopy of the suspended cells the low-resolution setup was employed in order to make use of the large working distance. The L929 cells in the transmission image are barely recognizable due to their fine-grained diffraction pattern next to the coarse patterned ice matrix (figure 1a). In the multiphoton 
autofluorescence image (figure 1b), the cells have considerable intensity contrast even without any staining. Cellular structures are discernible as a consequence of the horizontal optical slicing of multiphoton microscopy (slice thickness using the $0.5 \mathrm{NA}$ objective $\sim 5 \mu \mathrm{m})$. No nuclei are visible since the slice in figure $1 \mathrm{~b}$ was acquired near to the substrate surface. The dendritic channels between the ice grain interfaces in the medium also show a certain fluorescence due to the strong enrichment of weakly fluorescent medium compounds in the remaining fluid after ice formation. Due to the rapid cooling with rate of $100 \mathrm{~K} / \mathrm{min}$ in the presence of DMSO the morphology of the cells remains similar to the unfrozen state. Results of comparable resolution and contrast were obtained using the FLIM modality for fluorescence detection.

The use of intrinsic emitters in combination with long working distance optics makes this technique a suitable tool for cryo-storage quality control. For research purposes regarding physiologic responses of the cell upon the freezing procedure artificial functional fluorescent probes can be applied, providing a more differentiated observation. Since in particular the dynamics in the temperature range of specimen freezing are under investigation, the high resolution setup with immersion objective can be used (slice thickness using the 1.3 NA objective $\sim 1 \mu \mathrm{m}$ ). Figure 2 a illustrates the potential of multiphoton cryo-microscopy for dynamic studies with 
micrographs of adherent fluorescently labelled L929 cells before (left) and after (right) freezing under various conditions. All the micrographs clearly show cellular positions and morphology. Ice grain formation can be observed in detail, be it extracellular, intracellular or intranuclear. The effects of different freezing conditions, here with respect to DMSO content of the medium and cooling rate, on the ice grain number and shape are palpable. A formation of many small crystals in the cytoplasm and slightly larger crystals in the nucleus is found at high (but not vitrifying) cooling rate of $100 \mathrm{~K} / \mathrm{min}$ without cryo-protectant, as shown in the upper row. Moderate cooling rates of $10 \mathrm{~K} / \mathrm{min}$ under DMSO addition lead to the sporadic occurrence of very large intranuclear ice crystals (middle row) whereas at slow cooling under DMSO addition no ice formation can be optically resolved at all (bottom row). Correlating the intracellular ice formation behaviour to the preservation rate of vital functions will strongly encourage the understanding of different freezing injury mechanisms and the optimization of freezing protocols. A such differentiated observation technique will be of considerable impact in particular for reanimation practice of isolated organelles, like nuclei for nuclear transfer cloning [22], Since the Deleted: [22] destructive mechanism of ice formation is predominantly mechanical disruption of cellular structures, crystal formation as small and infrequent as possible will likely be favourable, herein achieved with 
$10 \%$ DMSO at a colling rate of $1 \mathrm{~K} / \mathrm{min}$. Adapted fluorescent reporter probes will open up ways to monitor physicochemical and biochemical changes during the freezing process and their role in cell damage.

Another important aspect is to discriminate between freezing-, storage- and thawing-injuries. A cell being intact after the freezing step may still be harmed during the cryo-storage or the thawing process, as shown in figure $2 \mathrm{~b}$. Several cells of the specimen are ruptured due to ice recrystallization in the course of thawing. Systematic investigations on the destructive mechanisms in cryostorage and their dependence on freezing parameters are in progress and will be published in future.

The intrinsic cellular fluorophores like $\mathrm{NAD}(\mathrm{P}) \mathrm{H}$ and flavins show no significant change in fluorescence efficiency upon freezing of the embedding medium, which makes these natural fluorescent stains suitable for investigations and control of the cellular morphology in cryogenic processes. Furthermore these compounds may be used as reporter probes for the cellular metabolism and redox state by means of more sophisticated fluorescence detection and analysis techniques, such as FLIM and spectral imaging in combination with an appropriate theoretical model (inter alia [23]). Deleted: [23] The use of intrinsic fluorophores makes this technique suitable for quality control purposes in cryo-banking. On the other hand, in most 
cases little is known about the number and nature as well as the molecular environments of the emitters in autofluorescence imaging. Functional imaging modalities as in particular FLIM are capable of mapping chemical and physical properties of the specimen but only, as long as the number and fluorescence properties of the different Deleted: quantitatively Deleted: if Deleted: emitters are known. So how can FLIM be applied expediently to autofluorescence imaging? A quantitative mapping of the specimen's chemical composition and physicochemical conditions is obviously not possible. One way to at least discriminate compartments of different composition or different physicochemical conditions is to phenomenlogically analyse the raw data without any anticipated model of fluorescence decay and to group similar decay behaviour from the image. This procedure is called global parameterisation and applied on the data from the same specimen as published in [24], Deleted: [24] The results are morphological images plus chemical discrimination of the compartments, but without information about the fluorescent species, their concentrations and environments. This method can be applied even without any information about the fluorophores. If some information about the main emitters is available, the raw data can be "forced" into a simplified decay model, neglecting contributions from secondary fluorophores. This method also does not yield exact compositions but tells which compartments are dominated by which major emitter. The potential of the latter analysis technique will be 
exemplarily demonstrated by using a leaf of the aquatic plant Elodea densa as specimen. The two major emitters in plant tissue are considered to be chlorophyll and lignin, all other fluorophores are disregarded from the calculation. In this experiment the low resolution setup because of its long working distance was used. Figure 3 shows the multiphoton fluorescence lifetime micrographs acquired with TCSPC at a subsurface depth of $20 \mu \mathrm{m}$ analysed according to the simplified model described by equation (1). The first column represents the specimen in a super-cooled state at $-20{ }^{\circ} \mathrm{C}$, the second column shows almost the same part of the leaf after ice formation within the plant tissue at $-80{ }^{\circ} \mathrm{C}$, visible as dark areas in the intercellular space. The displacement was due to the mechanical forces upon freezing. The early ice formation was extracellular and the heterogeneous distribution of chloroplasts indicates also an intracellular ice grain formation. The lifetime data are dominated by two discrete lifetime ranges: a long-lived component with a mean lifetime $\tau=1.3 \mathrm{~ns}$, which is found predominantly in the cell walls and vascular structures (probably lignin [25]) and a short-lived component with a mean lifetime of some hundred ps, which is predominantly found in the chloroplasts (probably chlorophyll [261[27]). Hence images for figure 3 were calculated applying a double exponential Deleted: [25] Deleted: [24] Deleted:

Deleted: [26]
Deleted: [25]
Deleted: [27]
Deleted: [26]
plus instrument response function (IRF) with fixed $\tau_{2}$ being $1.3 \mathrm{~ns}$ for 
lignin. The chlorophyll lifetime was freely fitted since this compound shows a very complex fluorescence decay [26][27]:

$$
\mathrm{F}(\mathrm{t})=\mathrm{IRF}+\mathrm{a}_{1} \cdot \exp \left(-\mathrm{t} / \tau_{1}\right)+\mathrm{a}_{2} \cdot \exp \left(-\mathrm{t} / \tau_{2}\right)
$$

The upper row of figure $3(a, b)$ shows the intensity images colour coded with the "chlorophyll-lifetime" $\tau_{1}$, the bottom row $(c, d)$ is colour coded with the "fraction of the lignin fluorescence" $a_{2}$ in percent. $a_{1}$ and $a_{2}$ may change upon cooling due to thermal variations of absortion and fluorescence properties of the emitters, while the actual chemical composition remains unchanged. Also $\tau_{1}$ may be altered in a complex way due to temperature dependent kinetics in the photophysics and photochemistry of chlorophyll and of the unidentified fluorescence background. Three major conclusions on the potential of FLIM for cryo-microscopy can be drawn from figure 3: (1) FLIM is generally applicable to frozen samples. We performed FLIM experiments down to liquid nitrogen temperatures., (2) FLIM may be used for the qualitative discrimination of endogenous emitters using simplified models as shown in the $\mathrm{a}_{2-}$ images in bottom row encoding the fraction of lignin emission (or by global parameterisation [24]) and (3) FLIM could be used as a readout technique for artificial functional lifetime probes at low temperatures as shown by the $\tau_{1}$-images in the upper row revealing variations in composition and location of the native fluorophores.
Deleted: endogeneous 
In conclusion, we found multiphoton cryo-microscopy to be a very promising tool in low temperature biotechnology, since it is capable of non-invasive 3D subcellular resolution with high contrast even in scattering media and can be used in situ. Hence physiological processes can be observed in real time together with the formation of ice crystals. This can already be achieved without artificial staining by utilizing native fluorescent compounds. Using customised exogenous probes, functional and chemical information become accessible, in particular by applying advanced fluorescence detection technology (e.g. FLIM and spectral imaging). These features make multiphoton cryo-microscopy a very feasible observation technique for both cryobiology research and cryostorage monitoring purposes. 


\section{Acknowledgements}

We thank Professor Karsten König, Universität des Saarlandes, for helpful discussions, Ms. Susan Zöllner for her assistance in cell culture and Dr. Stephen Shirley for critical review as regards content and language.

\section{Conflict of interest statement}

The authors have declared no conflict of interests. 


\section{References}

[1] Jain, J., Paulson, R., Oocyte cryopreservation 2006, Fertil. Steril. 86 , 1037-1046.

[2] Rebulla, P. et Lecchi, L., Cord blood banking and accreditation, ISBT Sci. Series 2007, 2, 91-95.

[3] Hunt, C.J., The Banking and Cryopreservation of Human Embryonic Stem Cells, Transfus. Med. Hemother. 2007, 34, 293-304.

[4] Benson B.E., Lane N., Benson E.E., Life in the frozen state, Formatted: Line spacing: Double Formatted: Bullets and Numbering Routledge, Chapman \& Hall, 2004.

[5] Mazur, P., The role of intracellular freezing in the death of cells cooled at supraoptimal rates, Cryobiol.1977, 14, 251-271.

[6] Mazur, A., Leibo, S.P., Chu, H.Y., A two-factor hypotheseis of freezing injury, Exp. Cell Res. 1973, 71, 345-355.

[7] Smith, D.J., Fahssi, W.M., Swanlund, D.J., Bischof, J.C., A parametric study of freezing injury in AT-1 rat prostate tumor cells, Cryobiol. 1999, 39, 13-28.

[8]_Frederickx, V., Michiels, A., Goossens, E., De Block, G., Van Steirteghem, A.C., Tournaye, H., Recovery, survival and functional evaluation by transplantation of frozen-thawed mouse germ cells, Human Reprod. 2004, 19, 948-953. 
[9] Takamatsu, H., Zawlodzka S., Contribution of extracellular ice formation and the solution effects to freezing injury of PC-3 cells suspended in $\mathrm{NaCl}$ solutions Cryobiol. 2006, 53, 1-11.

[10] Seki, S., Mazur, P., Kinetics and activation energy of recrystallization of intracellular ice in mouse oocytes subjected to interupted rapid cooling, Cryobiol. 2008, 56, 171-180.

[11] Bank, H. Mazur, P., Visualization of freezing damage, J. Cell Biol. $1973,57,729-742$.

[12] McClean, R.V., Holt, W.V., Johnston, S.D., Ultrastructural observations of cryoinjury in kangaroo spermatozoa, Cryobiol. 2007, $54,271-280$.

[13] Göppert, H.R., Ueber die Wärme-Entwicklung in den Pflanzen, deren Gefrieren und die Schutzmittel gegen dasselbe, Joseph Max \& Co., Breslau, 1830 (in German).

[14] Diller, K.R., Cravalho, E.G., A cryo-microscope for the study of freezing and thawing processes in biological cells, Cryobiology 1971, 7, 191-199.

[15] Ishiguro, H., Horimizu, T. Three-dimensional microscopic freezing and thawing behavior of biological tissues revealed by real-time imaging using confocal laser scanning microscopy, Int. J. Heat Mass Trans. 2008, in press. 
[16] Wang, X.F., Periasamy, A., Herman, B., Coleman, D.M., Fluorescence lifetime imaging: Instrumentation and application, Crit. Rev. Anal. Chem. 1992, 23, 369-395.

[17] Becker, W., Bergmann, A., Hink, M.A., König, K., Fluorescence lifetime imaging by time-correlated single-photon counting, Micr. Res. Tech. 2004, 63, 58-66.

[18]_König, K., Riemann, I., High-resolution multiphoton tomography of human skin with subcellular spatial resolution and picosecond time resolution, J. Biomed. Opt. 2003, 8, 432-439.

[19] Wang, X.F., Periasamy, A., Herman, B., Fluorescence lifetime imaging (FLIM): Instrumentation and applications, Crit. Rev. Anal. Chem. 1992, 23, 369-395.

[20] Scmacinski, H., Lakowicz, J.R., Fluorescence lifetime based sensing and imaging, Sens. Act. B 1995, 29, 16-24.

[21] Huang, S., Heikal, A.A., Webb, W.W., Two photon fluorescence spectroscopy and microscopy of $\mathrm{NAD}(\mathrm{P}) \mathrm{H}$ and flavoprotein, Biophys. J. 2002, 82, 2811-2825.

[22] Wakayama, S., Ohta, H., Hikichi, T., Mizutani, E., Production of healthy cloned mice from bodies frozen at $-20^{\circ} \mathrm{C}$ for 16 years, Proc. Natl. Acad. Sci. USA 2008, 105, 17318-17322.

[23] Bird, D.K., Yan, L., Vrotsos, K.M., Eliceiri, K. W., et. al., Metabolic mapping of MCF10A human breast cells via multiphoton 
fluorescence lifetime imaging of the coenzyme NADH, Canc. Res. $2005,65,8766-8673$.

[24] Stark, M., Dörr, D., Ehlers, A., et. al. Multiphoton imaging and fluorescence lifetime studies on unstained cells and tissues at cryogenic conditions, Proc. SPIE 2007, 6628, 662809-662834.

[25] Albinson, A., Li, S., Lundquist, K., Stomberg, R., The origin of lignin fluorescence, J. Mol. Struct. 1999, 508, 19-27.

[26] Krause, G.H., Weis, E., Chlorophyll fluorescence and photosynthesis: the basics, Annu. Rev. Plant Physiol. Plant Mol. Biol. 1991, 42, 313-349.

[27] Wendler, J., Holzwarth, A.R., State transitions in the green alga Scenedesmus obliquus probed by time resolved chlorophyll fluorescence spectroscopy and global data analysis, Biophys. J. $1987,52,717-728$. 


\section{Scheme \& Figure legends}

Scheme 1: A comparison of excitation and detection beampaths in confocal and multiphoton microscopy showingh why multiphoton is considerable less susceptible by sample scattering: (a) the NIR-excitation beam is much less scattered in biomaterials than the visible beam, (b) Also scattered fluorescence photons are detected in multiphoton microscopy because no confocal pinhole is required for depth resolution (horizontal slicing).

Figure 1: Cryo-preserved L929-cells under different imaging conditions: Image (a) shows a transmission micrograph and image (b) a multiphoton autofluorescence micrograph of nearly the same sample area (cells suspended in frozen culture medium with $5 \%$ DMSO as cryo-protectant at $\left.-40^{\circ} \mathrm{C}\right)$. The multiphoton fluorescence image was acquired with a laser wavelength of $760 \mathrm{~nm}$ and contrast adjusted. Freezing was carried out at a cooling rate of $-\Delta T / \Delta t=100 \mathrm{~K} / \mathrm{min}$.

Figure 2: Multiphoton microscopy of ice formation and recrystallization in the course of freezing and thawing of adherent L929 cell cultures. Extraand intracellular as well as extra- and intranuclear ice grains can be clearly discriminated. An excitation wavelength of $\lambda_{E X}=800 \mathrm{~nm}$ was used. Intensity images consist of overlays of the $390-465 \mathrm{~nm}$ channel (blue, 
Figure 3: Fluorescence lifetime micrographs of Elodea densa at $20 \mu \mathrm{m}$ subsurface depth at $\mathrm{T}=-20^{\circ} \mathrm{C}$ (left column) and $\mathrm{T}=-80{ }^{\circ} \mathrm{C}$ (right column). Raw images were acquired with $256 \times 256$ pixel $^{2}$ and interpolated to $512 \times 512$ pixel $^{2}$ for presentation. Pixels of less than $8 \%$ of the image maximum countrate are discarded from calculation. The upper row displays the short component lifetime $\tau_{1}$ from 150 (red) to $700 \mathrm{~ns}$ (blue) in a RGB-color-code, highlighting the chloroplasts against the intracellular background. The bottom row shows the "fraction of lignin fluorescence" $a_{2}$ from $0 \%$ (red) to $100 \%$ (blue) highlighting the cell walls and vascular 
structures. At $-20{ }^{\circ} \mathrm{C}$ yet no ice formed inside the plant, at $-80{ }^{\circ} \mathrm{C}$ ice formation in the extracellular matrix took place, visible as dark areas. The micrographs were acquired with a excitation wavelength of $\lambda_{E X}=780 \mathrm{~nm}$. The specimen was frozen with a cooling rate of $-\Delta T / \Delta t=10 \mathrm{~K} / \mathrm{min}$ in its natural medium (aquaristic water). 


1
2
3
4
5
6
7
8
9
10
11
12
13
14
15
16
17
18
19
20
21
22
23
24
25
26
27
28
29
30
31
32
33
34
35
36
37
38
39
40
41
42
43
40
45
49
50
50
51
53
55
50

a) excitation beampath

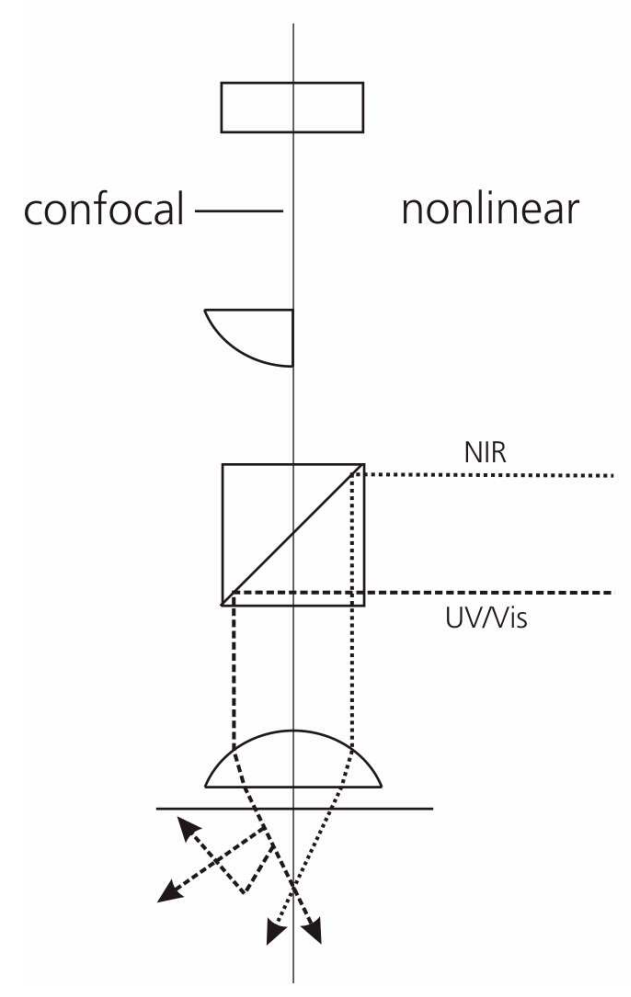

b) detection beampath

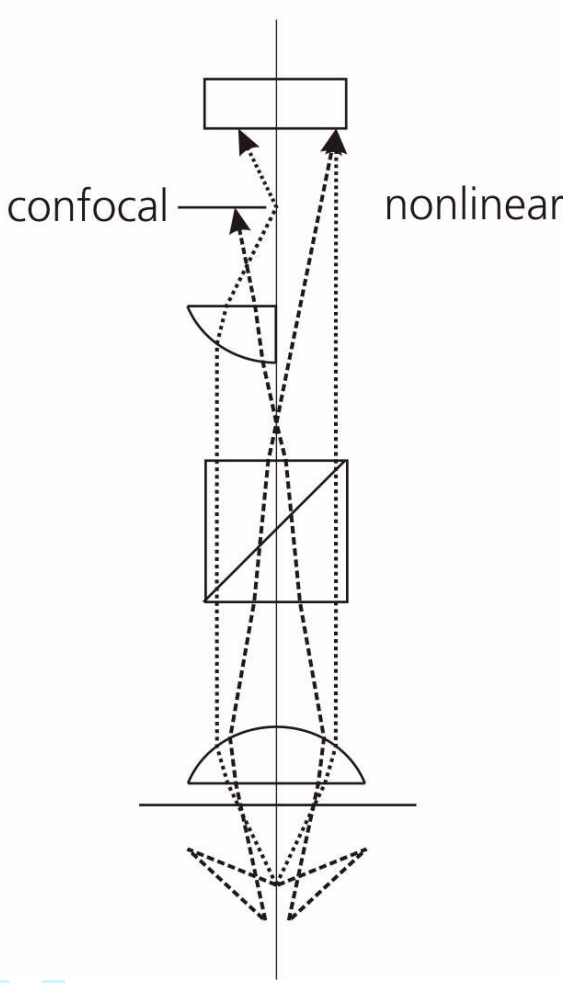



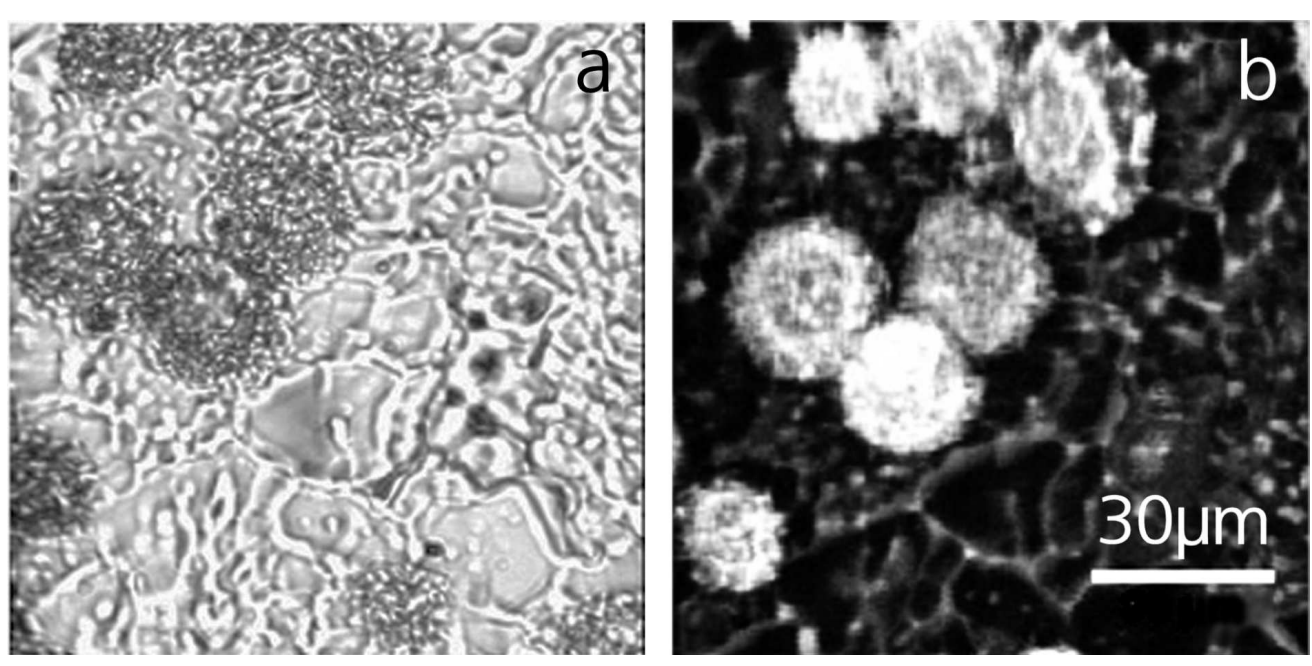

$160 \times 77 \mathrm{~mm}(300 \times 300$ DPI $)$

Wiley-VCH 

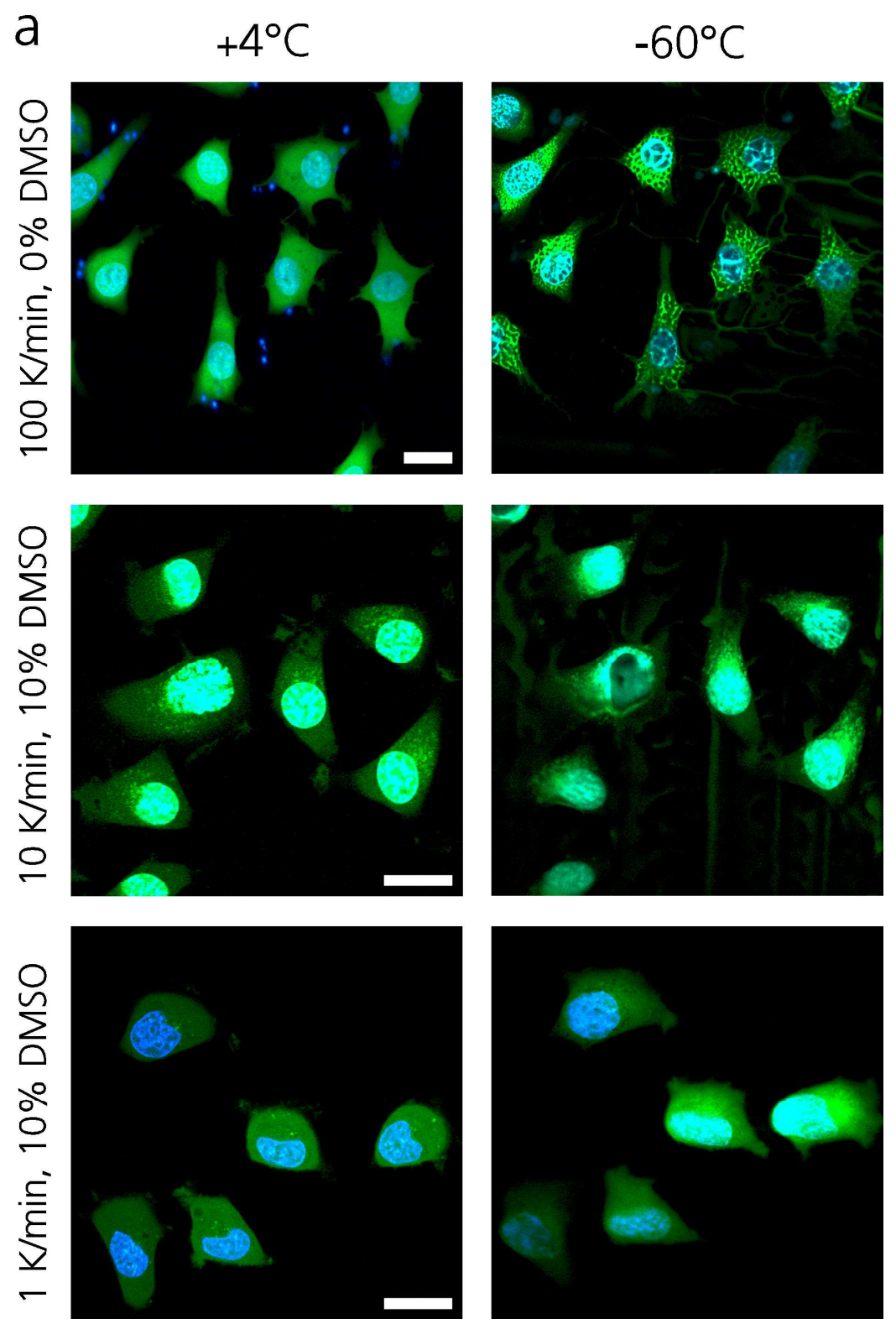

$160 \times 238 \mathrm{~mm}(300 \times 300 \mathrm{DPI})$ 
b
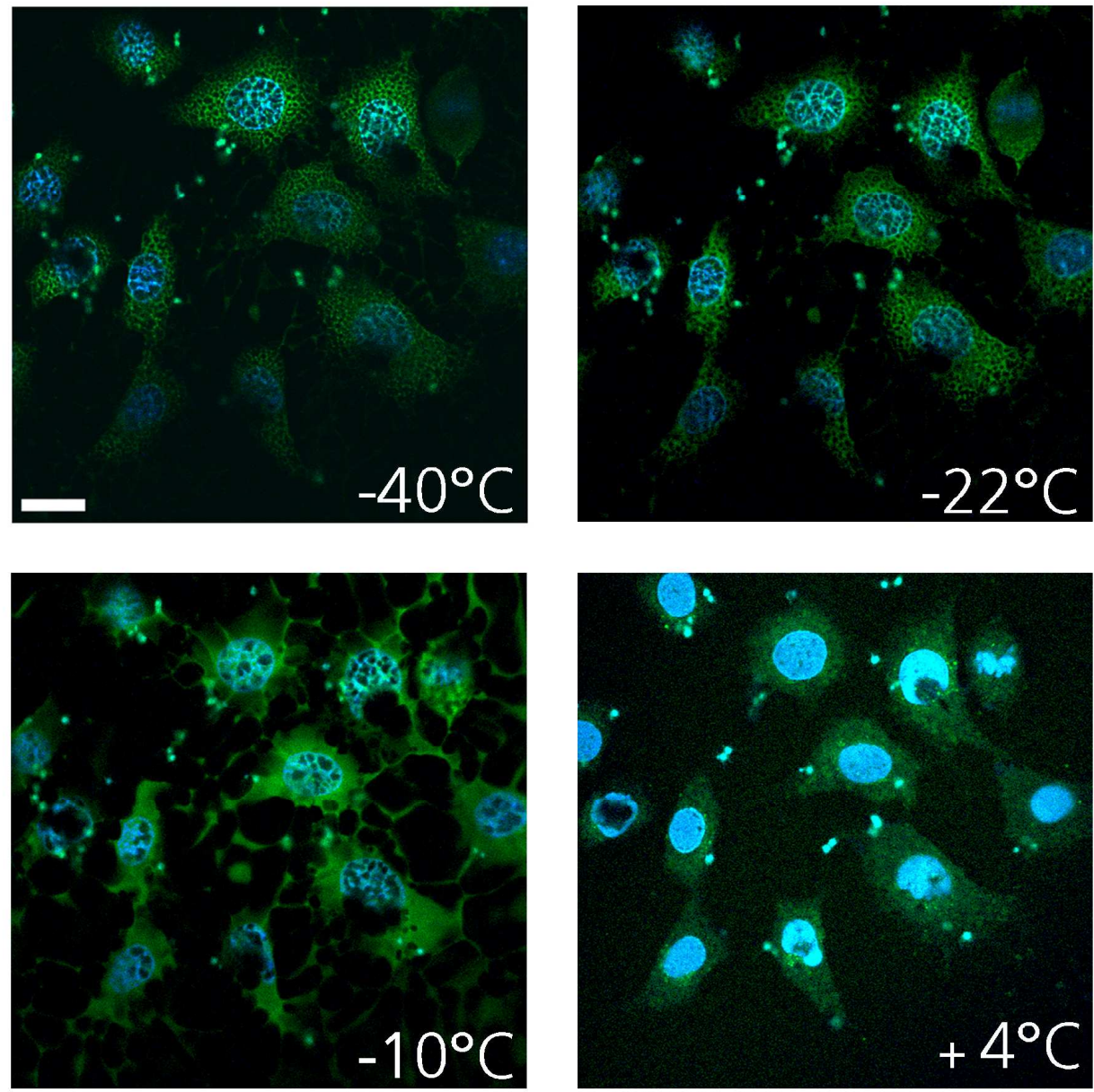

$160 \times 165 \mathrm{~mm}(300 \times 300 \mathrm{DPI})$

Wiley-VCH 


\section{Page 29 of 29}

Biotechnology Journal
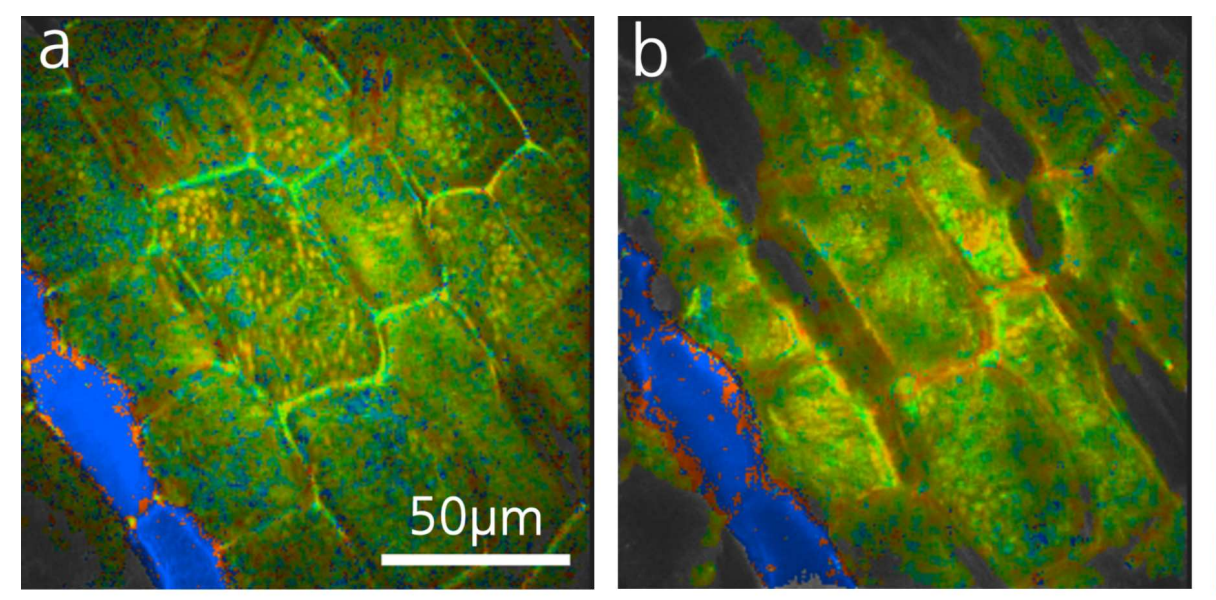

700
600

$\stackrel{2}{\equiv}$

250

150
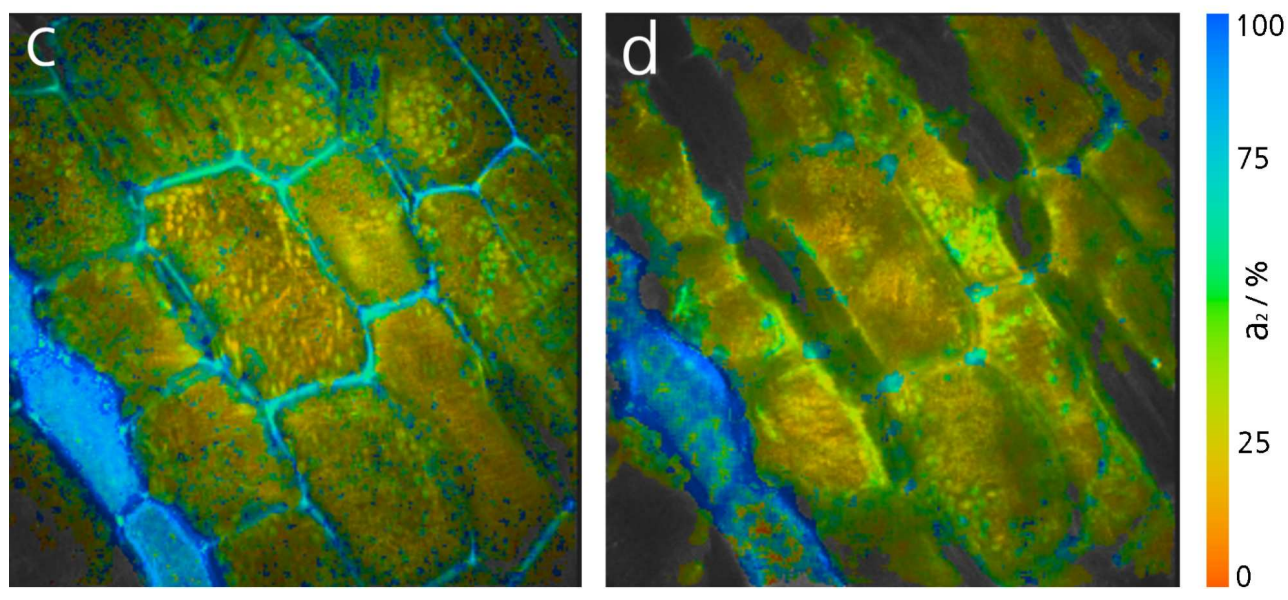

$160 \times 143 \mathrm{~mm}(320 \times 320$ DPI $)$ 